\title{
Correction to: A study for multi-layer skin burn injuries based on DPL bioheat model
}

\author{
Rajneesh Kumar Chaudhary ${ }^{1}$ Kabindra Nath Rai ${ }^{2} \cdot$ Jitendra Singh ${ }^{1}$
}

Published online: 11 September 2020

○) Akadémiai Kiadó, Budapest, Hungary 2020

\section{Correction to: Journal of Thermal Analysis and Calorimetry https://doi.org/10.1007/s10973-020-09967-3}

In the original publication of the article, the following equations has been incorrectly published. The corrected equations are given below:

$Q_{\mathrm{d}}=\frac{D_{f} c_{\rho \mathrm{W}}\left(\rho_{\mathrm{s}}-\rho_{\mathrm{c}}\right)}{(\Delta r)^{2}}\left(T(r, t)-T_{0}\right)$,

$\Delta m=\frac{D_{v} M_{\mathrm{W}}}{R_{\mathrm{a}} \delta c}\left[\left(\frac{P_{\mathrm{W}}}{T_{\mathrm{W}}}\right)_{\mathrm{s}}-\left(\frac{P_{\mathrm{W}}}{T_{\mathrm{W}}}\right)_{a} R H\right]$,

$M=M_{l}\left\{\begin{array}{l}l=1 \text { for first kind non-Fourier boundary condition, } \\ l=2 \text { for second kind non-Fourier boundary condition, } \\ l=3 \text { for third kind non-Fourier boundary condition. }\end{array}\right.$

$N_{1}=\left[P_{\mathrm{mo}}-Q_{\mathrm{vo}}+\frac{F_{l}\left(F_{\mathrm{o}}\right)}{h^{2}} P_{\mathrm{mo}}-Q_{\mathrm{vo}} \cdots P_{\mathrm{mo}}-Q_{\mathrm{vo}}\right]^{\mathrm{T}}$,

$\psi\left(F_{\mathrm{o}}\right)=\left[\begin{array}{ccccccccc}\psi_{10} & \psi_{11} & \cdots & \psi_{1 \mathrm{M}^{\prime}-1} & \psi_{20} & \psi_{21} & \cdots & \psi_{2 \mathrm{M}^{\prime}-1} & \psi_{2^{k-1} 0} \\ & \psi_{2^{\mathrm{k}-1}} & \cdots & \psi_{2^{k-1} \mathrm{M}^{\prime}-1} & & & & \end{array}\right]^{\mathrm{T}}$

The original article can be found online at https://doi.org/10.1007/ s10973-020-09967-3.

Jitendra Singh

jitendraprof@gmail.com

Rajneesh Kumar Chaudhary

kingrajneesh222kumar@gmail.com

Kabindra Nath Rai

knrai.apm@itbhu.ac.in

1 Department of Mathematics, Institute of Science, Banaras Hindu University, Varanasi 221005, India

2 Department of Mathematical Sciences, IIT-BHU, Varanasi 221005, India
$C_{2}^{\mathrm{T}}-M_{l} C_{2}^{\mathrm{T}} X_{2}-Z_{2}=0$,

$$
\begin{aligned}
& \theta\left(x, F_{\mathrm{o}}\right)=\frac{1}{U_{1,1}\left(U_{1,2}+1\right) V_{1}}\left[-\left(U_{1,2}+U_{1,3}\right) Q_{\mathrm{do}} \theta_{\mathrm{w}}\right. \\
& \quad+P_{\mathrm{mo}}\left(\alpha\left(U_{1,2}+U_{1,3}\right) \theta_{\mathrm{w}}+\left(U_{1,1}-1\right)\left(U_{1,1}-U_{1,2}\right)\right) \\
& \left.\quad+\left(U_{1,2}-U_{1,1}\right)\left(U_{1,1}-1\right) Q_{\mathrm{vo}}\right] \\
& \quad+\sum_{\mathrm{n}=0}^{\infty}\left(\frac{1}{U_{1,4} V_{1, \mathrm{n}}}\right)\left[e ^ { \mathrm { F } _ { \mathrm { o } } \mathrm { V } _ { 1 , \mathrm { n } } - \mathrm { U } _ { 1 , 4 } ( x + 2 ) } \left(\left(e^{2 \mathrm{U}_{1,4} x}+e^{2 \mathrm{U}_{1,4}}\right) \theta_{\mathrm{w}}\left(Q_{\mathrm{do}}+V_{1, \mathrm{n}}\right)\right.\right. \\
& \quad+P_{\mathrm{mo}}\left(\left(e^{2 \mathrm{U}_{1,4}}-e^{\mathrm{U}_{1,4} x}\right)\left(e^{\mathrm{U}_{1,4} x}-1\right)-\alpha\left(e^{2 \mathrm{U}_{1,4} x}+e^{2 U_{1,4}}\right) \theta_{\mathrm{w}}\right) \\
& \left.\left.\quad+Q_{\mathrm{vo}}\left(-\left(e^{2 \mathrm{U}_{1,4}}-e^{\mathrm{U}_{1,4} x}\right)\right)\left(e^{\mathrm{U}_{1,4} x}-1\right)\right)\right],
\end{aligned}
$$

$$
\begin{aligned}
\theta\left(x, F_{\mathrm{o}}\right)= & -\frac{1}{U_{2,1}\left(U_{2,2}+1\right) V_{2}}\left[\left(U_{2,2}+U_{2,3}\right) Q_{\mathrm{do}} \theta_{\mathrm{w}}\right. \\
& +P_{\mathrm{f}}^{2}\left(\left(U_{2,2}-U_{2,1}\right)\left(-1+U_{2,1}\right) \theta_{\mathrm{b}}+\left(U_{2,2}+U_{2,3}\right) \theta_{\mathrm{w}}\right) \\
& \left.+P_{\mathrm{mo}}\left(\left(U_{2,2}-U_{2,1}\right)\left(-1+U_{2,1}\right)-\left(U_{2,2}+U_{2,3}\right) \alpha \theta w\right)\right] \\
& +\sum_{\mathrm{n}=0}^{\infty} \frac{1}{U_{2,4} V_{2, n}}\left[e ^ { \mathrm { F } _ { \mathrm { o } } V _ { 2 , \mathrm { n } } - U _ { 2 , 4 } ( x + 2 ) } \left(\left(e^{2 U_{2,4} x}+e^{2 U_{2,4}}\right)\right.\right. \\
& \theta_{\mathrm{w}}\left(Q_{\mathrm{do}}+V_{2, \mathrm{n}}\right)+P_{\mathrm{f}}^{2}\left(\left(e^{2 U_{2,4}}-e^{U_{2,4} x}\right)\left(e^{U_{2,4} x}-1\right) \theta_{\mathrm{b}}\right. \\
& \left.+\left(e^{2 U_{2,4} x}+e^{2 U_{2,4}}\right) \theta_{\mathrm{w}}\right)+P_{\mathrm{mo}}\left(\left(e^{2 U_{2,4}}-e^{U_{2,4} x}\right)\right. \\
& \left.\left.\left.\left(e^{U_{2,4} x}-1\right)-\left(e^{2 U_{2,4} x}+e^{2 U_{2,4}}\right) \alpha \theta_{\mathrm{w}}\right)\right)\right],
\end{aligned}
$$

$$
\begin{aligned}
& \theta\left(x, F_{\mathrm{o}}\right)=-\frac{1}{U_{3,1}\left(U_{3,2}+1\right) V_{3}} \\
& \quad\left[\left(U_{3,2}+U_{3,3}\right) Q_{\mathrm{do}} \theta_{\mathrm{w}}+P_{\mathrm{f}}^{2}\left(\left(U_{3,2}-U_{3,1}\right)\left(-1+U_{3,1}\right) \theta_{\mathrm{b}}\right.\right. \\
& \left.\quad+\left(U_{3,2}+U_{3,3}\right) \theta_{\mathrm{w}}\right) \\
& \left.\quad+P_{\mathrm{mo}}\left(\left(U_{3,2}-U_{3,1}\right)\left(-1+U_{3,1}\right)-\left(U_{3,2}+U_{3,3}\right) \alpha \theta w\right)\right] \\
& \quad+\sum_{\mathrm{n}=0}^{\infty} \frac{1}{U_{3,4} V_{3, \mathrm{n}}}\left[e ^ { \mathrm { F } _ { \mathrm { o } } V _ { 3 , \mathrm { n } } - U _ { 3 , 4 } ( x + 2 ) } \left(\left(e^{2 U_{3,4} x}+e^{2 U_{3,4}}\right)\right.\right. \\
& \quad \theta_{\mathrm{w}}\left(Q_{\mathrm{do}}+V_{3, \mathrm{n}}\right)+P_{\mathrm{f}}^{2}\left(\left(e^{2 U_{3,4}}-\mathrm{e}^{U_{3,4} x}\right)\left(e^{U_{3,4} x}-1\right) \theta_{\mathrm{b}}\right. \\
& \left.\quad+\left(e^{2 U_{3,4} x}+e^{2 U_{3,4}}\right) \theta_{\mathrm{w}}\right) \\
& \quad+P_{\mathrm{mo}}\left(\left(e^{2 U_{3,4}}-e^{U_{3,4} x}\right)\left(e^{U_{3,4} x}-1\right)\right. \\
& \left.\left.\left.\quad-\left(e^{2 U_{3,4} x}+e^{2 U_{3,4}}\right) \alpha \theta_{\mathrm{w}}\right)\right)\right],
\end{aligned}
$$


The word "Temperature" should be omitted in the figure captions 5 to 13 . The corrected figure captions are given below:

Fig. 5 Epidermis layer: Effect of $F_{\text {oq }}=0.00696379$ and $F_{\text {ot }}=0$ on skin temperature with the first kind non-Fourier boundary condition

Fig. 6 Dermis layer: Effect of $F_{\text {oq }}=0.0140766$ and $F_{\text {ot }}=0$ on skin temperature with the first kind non-Fourier boundary condition

Fig. 7 Subcutaneous layer: Effect of $F_{\text {oq }}=0.00791667$ and $F_{\text {ot }}=0$ on skin temperature with the first kind nonFourier boundary condition

Fig. 8 Epidermis layer: Effect of $F_{\text {oq }}=F_{\text {ot }}=0.00696379$ on skin temperature with the first kind non-Fourier boundary condition

Fig. 9 Dermis layer: Effect of $F_{\text {oq }}=F_{\text {ot }}=0.0140766$ on skin temperature with the first kind non-Fourier boundary condition

Fig. 10 Subcutaneous layer: Effect of $F_{\text {oq }}=F_{\text {ot }}=0.00791667$ on skin temperature with the first kind non-Fourier boundary condition
Fig. 11 Epidermis layer: Effect of lagging on skin temperature with the first kind non-Fourier boundary condition at $F_{\mathrm{o}}=0.5$

Fig. 12 Dermis layer: Effect of lagging on skin temperature with the first kind non-Fourier boundary condition at $F_{\mathrm{o}}=0.5$

Fig. 13 Subcutaneous layer: Effect of lagging on skin temperature with the first kind non-Fourier boundary condition at $F_{\mathrm{o}}=0.5$

The original article has been corrected.

Publisher's Note Springer Nature remains neutral with regard to jurisdictional claims in published maps and institutional affiliations. 